\title{
Li-Fi Synchronicity towards 5 G: concepts, challenges and opportunities
}

Shilpi Sharma

Amity University, Uttar Pradesh, India

Email: ssharma22@amity.edu

\begin{abstract}
It is quite frustrating when the moderate speed of system prompts restricts availability and long handling hours while utilizing remote web either at home system or coffeehouse or airplane terminal or going after data transfer capacity at a meeting. As more clients are tapped in with their gadgets, the stopped up wireless transmissions make it hard to hook on a dependable sign. Radio wave is by all accounts completely abused and other range should also be investigated. For this reason, fibre optics have been utilized to send information through LED light. D-Light can deliver information rates speedier than 10 super bits for each second, which is far quicker than normal broadband association.
\end{abstract}

Keywords—Light Fidelity, Light Emitting diode, Line of Sight,Remotely Operated Vehicles

\section{INTRODUCTION}

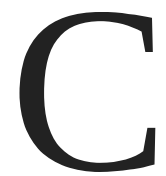

ommunication, the most rising part in everyone's life is through trading data either on wired or remote gadgets. WiFi is the most adaptable and powerful innovation which reduced with radio/microwave frequencies for information transmission. The accessibility of high affectability beneficiaries, that gives wide scope at low frequencies and LOS communication at high frequencies. Because of steadily expanding stipulation for remote communication, Wi-Fi is confronting numerous difficulties to be specific, limit, accessibility, proficiency and security.[1] Li-Fi is a remote correspondence framework in which light is utilized as a bearer signal rather than customary radio recurrence as in Wi-Fi. Li-Fi is an innovation that uses light radiating diodes to transmit information remotely. VLC utilizes quick beats of light to transmit data remotely that can't be recognized by human eye.[11]. The invaluable thing is the remote correspondence which diminishes the expense enormously.[2].Both Li-fi and $\mathrm{Wi}-\mathrm{Fi}$ utilizes electromagnetic range for information transmission, however though Wi-Fi uses radio waves, Li-Fi utilizes noticeable light correspondence as a part of the scope of 100Mbps. To correct this constraint of Wi-Fi in little scope territory, WiMax has been presented. WiMAX is a remote correspondences standard intended to give 30 to 40 megabit-per-second information rates, with the 2011 overhaul giving up to $1 \mathrm{Gbit} / \mathrm{s}$ for altered stations. As velocity of light is path quicker than radio waves consequently it can be utilized with a rate of around 250 times more than any rapid broadband and its pace is over the $1 \mathrm{Gbps}$.

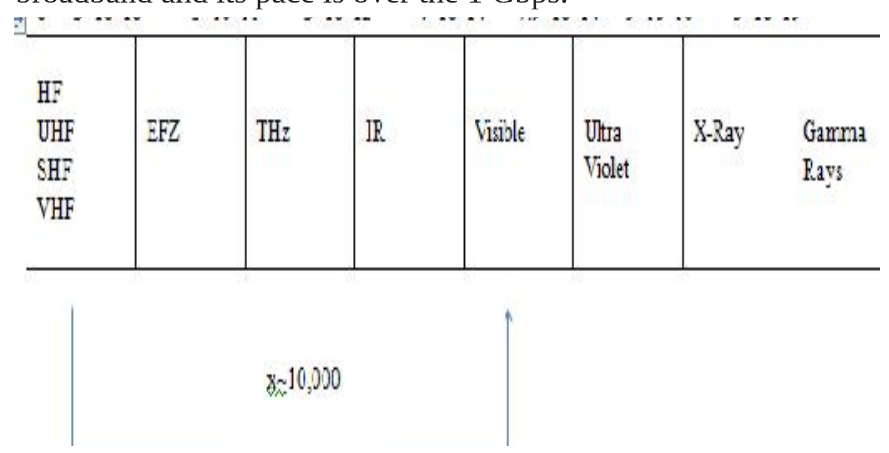

Figure 1: Electromagnetic Spectrum ranging from $0-3 * 10 \wedge 19$.

\section{RELATED WORK}

$\mathrm{Li}-\mathrm{Fi}$ is transmission of information through illumination so as to brighten the fibre out of fibre optics by sending information through a LED light that differs in power speedier than the human eye can follow. Li-Fi is the term used to name the quick and shabby remote correspondence framework, which is the optical adaptation of Wi-Fi. The term was initially utilized as a part of this setting by Herald Haas in his TED Global chat on Visible Light Communication. "At the heart of this innovation is another era of high splendour lightdischarging diodes", says Herald Haas from the 
University of Edinburgh, UK. Li-Fi, as it has been named, has as of now accomplished blisteringly rapid in the lab. Specialists at the Heinrich Hertz Institute in Berlin, Germany, have achieved information rates of more than 500 megabytes for each second utilizing a standard white-light LED. In October 2011 various organizations and industry bunches shaped the Li-Fi Consortium, to advance rapid optical remote frameworks and to beat the constrained measure of radio based remote range accessible by misusing a totally distinctive part of the electromagnetic range. The innovation was shown at the 2012 Consumer Electronics Show in Las Vegas utilizing a couple of Casio advanced cells to trade information utilizing light of fluctuating power emitted from their screens, perceivable at a separation of up to ten meters. The consortium trusts it is conceivable to accomplish more than 10 Gbps, hypothetically permitting a top notch film to be downloaded in 30 seconds. This latest model was made with pure $\mathrm{LiFi}$, the University of Edinburgh twist out light correspondences innovation organization that leads the business sector in item research and commercialisation of Li-Fi.

\section{WORKING}

This innovation depends on the VLC which utilizes the noticeable light for information communication . In VLC, we utilize a source of illumination which can deliver illumination as well as send data utilizing the same light. VLC is illumination alongside communication.[19]. It is actualized by utilizing a light at the downlink transmitter. Ordinarily the light gleams at a consistent current supply however quick and inconspicuous varieties in current can be made to deliver the optical output.[9].The LEDs can be exchanged on and off rapidly which gives the clients the open doors for transmitting information since working rate of a LED is under $1 \mu \mathrm{s}$. The rate at which the LED's flicker should be differed relying on the information that should be transmitted. This undetectable on-off action empowers a sort of information transmission utilizing parallel codes. A light delicate gadget (a photo detector) gets the sign and changes over it again into unique data.[18]. Right when a predictable current is associated with a LED light a constant flow of photons are transmitted from the knob which is seen as visible light. The yield of light reduces, if the current is moved bit by bit. Since LED bulbs are semi-conducting-devices , the optical yield parallel to current, can be directed at to a great degree high speed which can be perceived by a photo identifier device and changed over back to electrical current. The force change is immaterial to the human eye, and as needs be communication is basically as steady as RF. Using this system, quick information can be transmitted from a LED light.

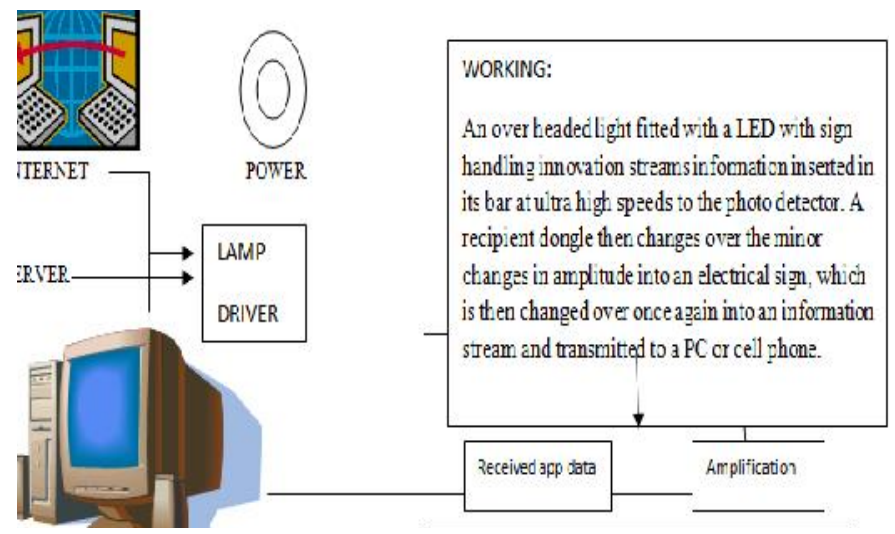

Figure 2: Working of LiFi

\section{APPLICATIONS}

There are various utilizations of this innovation, from open web access through road lights to autosteered autos that convey through their headlights. Utilizations of Li-Fi can reach out in regions where the Wi-Fi innovation does not have its vicinity like therapeutic innovation, power plants and different other areas.[8].

- $\quad$ Education System: LI-FI can supplant WIFI in instructive foundations and give quicker web speeds. Even the general population can make utilization at the same rate as has been assigned.

- Therapeutic Applications: WI-FI is not permitted operation theaters since they can meddle with restorative supplies. Also, their radiations posture dangers for patients. LI-FI utilizes light and consequently can be utilized instead of WI-FI.[9]. 
- Web access in flying machines: The utilization of WI-FI is precluded inside planes since they can meddle with the navigational frameworks of the plane. The clients access low speed web at high rates. Therefore, LI-FI is a protected distinct option for WI-FI in air ships since it utilizes light and can give speedier web access.[9].

- Underwater applications: Underwater ROVs work from substantial links that supply their energy and permit them to get signals from their pilots above. Be that as it may, the tie utilized as a part of ROVs is not sufficiently long to permit them to investigate bigger ranges. In the event that their wires were supplanted with light say from a submerged, powerful light then they would be much more liberated to investigate. They could likewise utilize their headlamps to communicate with each other, handling information independently and sending their discoveries occasionally back to the surface.[8].

- Natural Crisis Management: In times of regular catastrophes such seismic tremors, LI-FI can be utilized as an effective method for communication since it utilizes light which unlike RF is not blocked by dividers or other such things.

- Radio show: a lot of force is required by radio poles keeping in mind the end goal to telecast and this makes them very wasteful. LEDs then again require low energy to work and this implies LI-FI additionally utilizes almost no power.

- $\quad$ Sun based boards: Solar boards on houses or different items can be adjusted to additionally be utilized for portable remote correspondence.

\section{FEATURES}

\begin{tabular}{|c|c|}
\hline mairs : vi Light Fidetity & 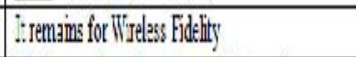 \\
\hline $\begin{array}{l}\text { transmits itrumation utilizg light with the } \\
\text { stanne of LED b. Jbs. }\end{array}$ & 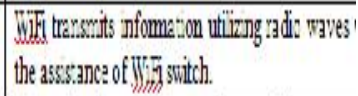 \\
\hline $\begin{array}{l}\text { not have any in:aference ssuls the nado } \\
\text { luance waves. }\end{array}$ & $\begin{array}{l}\text { Have irterfezmce isules from adjacent as } \\
\text { poicts'suters) }\end{array}$ \\
\hline aspresent [LDA cunsisten: devicas & 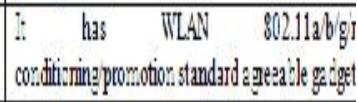 \\
\hline 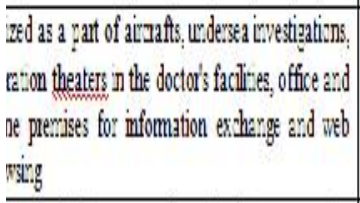 & 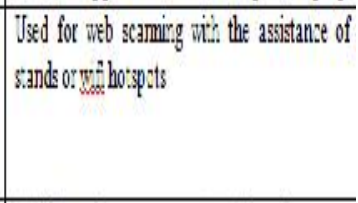 \\
\hline $\begin{array}{l}\text { tuction is lass, ce:s go trousgh sally ceean water; } \\
\text { bis dengy ragon }\end{array}$ & 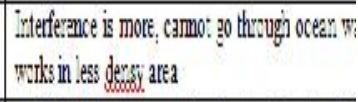 \\
\hline 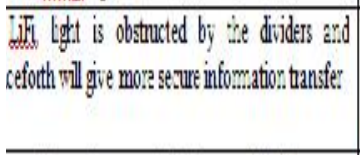 & 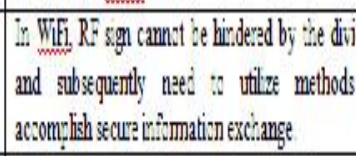 \\
\hline 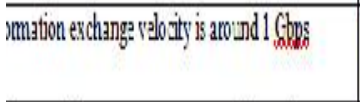 & 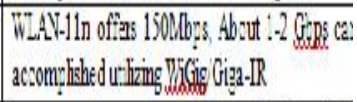 \\
\hline houssnd times recumerce ange of the adio & 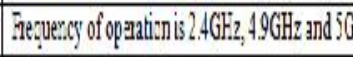 \\
\hline isin higg: tick envirument & $\begin{array}{l}\text { Wouks in less this exinomert berause } \\
\text { obstuction related isses }\end{array}$ \\
\hline paseraracon s arourd 1/ meters & 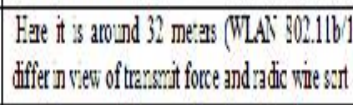 \\
\hline 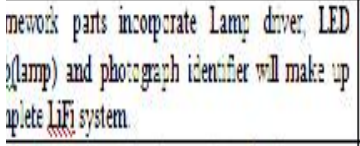 & 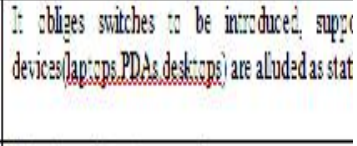 \\
\hline Imation tansmising is & \\
\hline
\end{tabular}

Table 1: Comparisons between LiFi and WiFi on the basis of their features. 


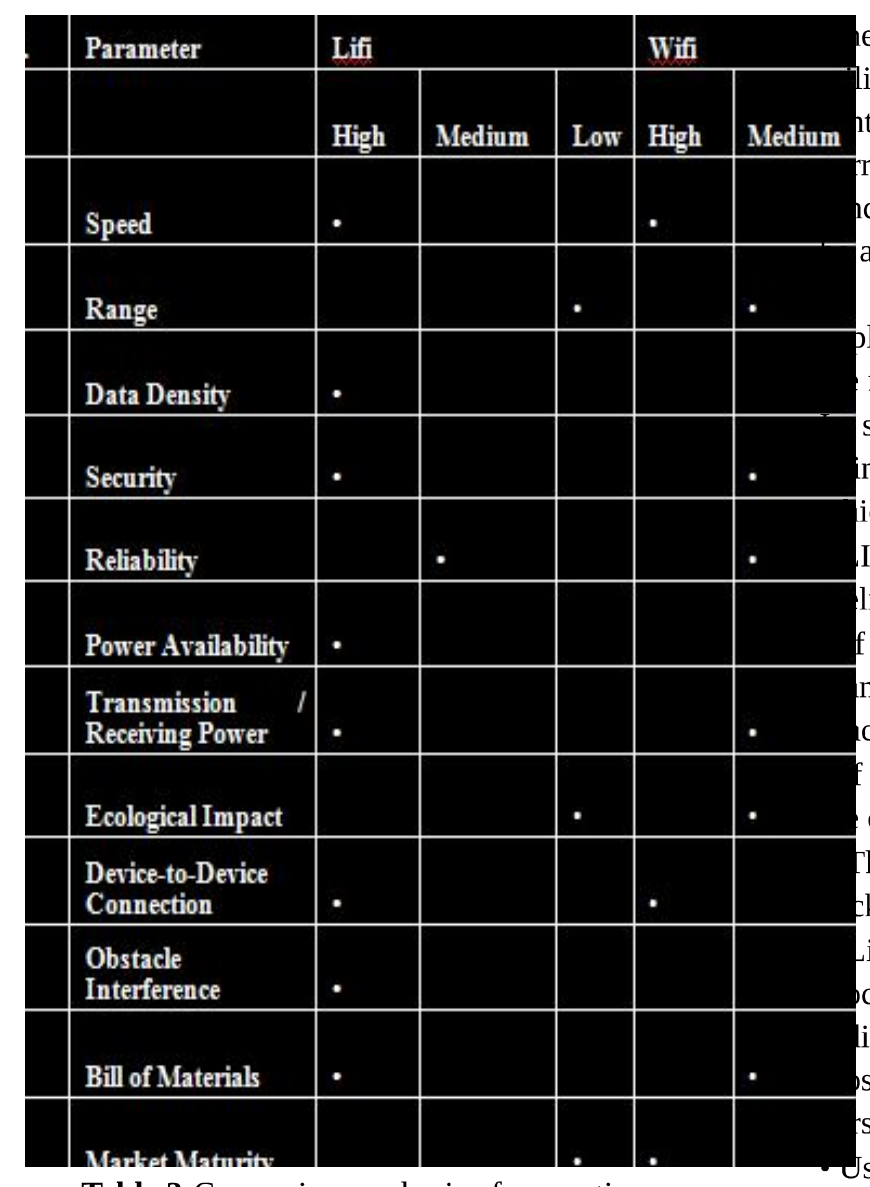

Table 2:Comparison on basis of properties.

\section{FUTURE SCOPE AND LIMITATIONS}

The region of $\mathrm{Li}-\mathrm{Fi}$ is exceptionally wide in the way of Hospitals, Academics, Airlines and the sky is the limit from there. It can be utilized as a part of the spots where it is hard to lay the optical fiber. Utilizing $\mathrm{Li}-\mathrm{Fi}$, each globule can be utilized like a Wi-Fi hotspot to transmit remote information and it will continue towards cleaner, greener, more secure and brighter remote future. The idea of Li-Fi is a honest to goodness and extremely effective backing to radio-based remote. It can be utilized as a part of petroleum or substance plants where other transmission or frequencies could be hazardous. [15].

With this improved innovation, individuals can get to remote information with the LED's introduced on the go at high rates. In different military applications, where RF based correspondences are not permitted, Li-Fi could be a practical distinct option for safely pass information at high rates to er military vehicles. Additionally LEDs can be ized viably to do VLC in numerous healing ter applications where RF based respondences could be conceivably hazardous. ce light can't infiltrate through dividers, it could a restriction to this innovation. By and by, given high rates of information transmission and lications in numerous fields, Li-Fi is certainly future in remote communication.[11] spite of the fact that there are a ton of focal nts of LI-FI, there are still sure difficulties ich should be succeed.

I-FI requires Line of Sight or range to be full of ling.

the device is set up outside, it would need to nage changing climate conditions and it is not ctical to utilize LEDs in sunshine.

the device is set up inside, one would not have capacity to move the collector.

he issue of how the beneficiary will transmit $\mathrm{k}$ to the transmitter still continues.

ight waves can without much of a stretch be cked and can't infiltrate thick dividers like the io waves and noticeable light can't enter through st dividers and is effortlessly obstructed by some son basically strolling before the LED source.

sers get to be reliant on the light hotspot for web access which can't be promptly accessible all over and at whatever time. In the event that the light source glitches, clients lose access to the web. Still there are a few backgrounds like it can just transmit when in the observable pathway.

As the usage of LED increases so is the possibility of LiFi. The affect is direct and it is one of the most crucial factors in determining the future of LiFi. 


\section{UIUWLII UI LED DUSIIIESS III IIIUId}

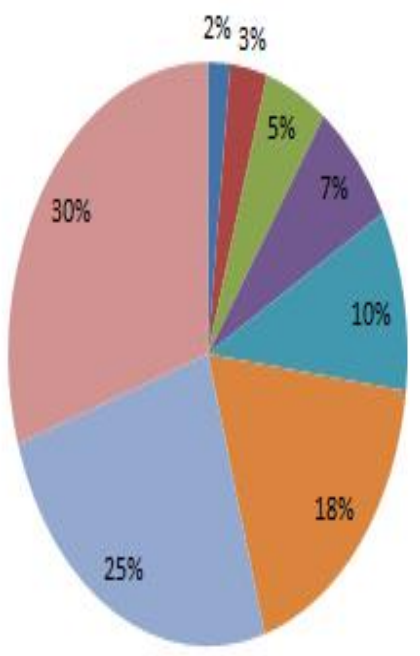

Figure 3: Growth of LED in India

\section{RESULT}

Scientists are creating micron estimated LEDs which glimmer on and off 1000 times quicker than bigger LEDs. They give quicker information exchange furthermore consume up less room. Harald Haas' gathering, with scientists from Universities of Oxford, Cambridge, Strathclyde and St. Andrews, are included in a four-year, £5.8 million undertaking subsidized by the Engineering and Physical Sciences Research Council. They are examining ultra-parallel VLC, which makes utilization of different hues to offer high-data transfer capacity connecting more than few meters. The Lifi Consortium has built up a superior LED which can give information rates near 4 Gbps while working just on 5 milli-watts of optical yield power and making utilization of high-transfer speed photodiodes at the less than desirable end. By improving the separation, utilizing a straightforward lens, they can send information a separation of 10 meters at velocities of $1.1 \mathrm{Gbps}$. As of now, the University of Edinburgh records the accompanying ventures at present in advancement:

1) Optical Multiuser MIMO - It includes misusing the certainties that LEDs offer extremely directional pillars and that power regulation (IM) does not experience the ill effects of multipath blurring. The need is to find new theories for multiuser, organized Li-Fi frameworks.
2) Interference Management in Cellular $\mathrm{Li}-\mathrm{Fi}$ Networks - It is coordinated towards creating obstruction cancelation methods particular to Li-Fi. The undertaking additionally thinks about cell a collaboration and impedance shirking methods.

a 2 3) The Internet of Things - This depends on the a2 way that because of the cheap way of photo a detectors and LEDs, it is conceivable to grow little and low-complex handset units that permit any a LED light to go about as a rapid information a transmitter.

2 4) Li-Fi Spatial Modulation - This is another in advanced adjustment and MIMO strategy which takes into consideration exceptionally vitality productive transmitters since it just needs a solitary transmitter chain. The venture investigates how spatial balance could be utilized to bolster darkening of light in Li-Fi frameworks and the effect of lenses and polarisers on the execution of optical spatial regulation.

5) Novel Digital Modulation Techniques for Li-Fi The advanced tweak systems are obliged by the way that signs must be genuine esteemed and positive since Li-Fi utilizes direct discovery and force adjustment. These imperatives cause misfortunes in range and influence proficiency. This anticipate endeavours to conquer these constraints by growing new Li-Fi tweak strategies, for example, Orthogonal Frequency Division Multiplexing (OFDM), Carrier-less Amplitude Modulation (CAP) and Pulse-Amplitude

Modulation.

6) Self-Powered Li-Fi - This anticipate takes a gander at vitality gathering ideas alongside vitality effective handset innovations for Li-Fi frameworks and requires calculations of low computational intricacy and also vitality productive methods for computerized balance. New circuit plans and new synchronization and MAC methods fall inside the extent of this anticipate.

Further research in the field can look into the following issues:

1) Driving illumination grade LEDs at high speed

2) Increasing data rate with parallelism/arrays

3) Achieving low complexity/low cost modulation

4) Overcoming the line of sight constraint 
5) Achieving seamless interoperability with other networks

6) Making Li-Fi work in environments with little or no light

Are you aware that lifi technology is $82 \%$ faster than other technologies?

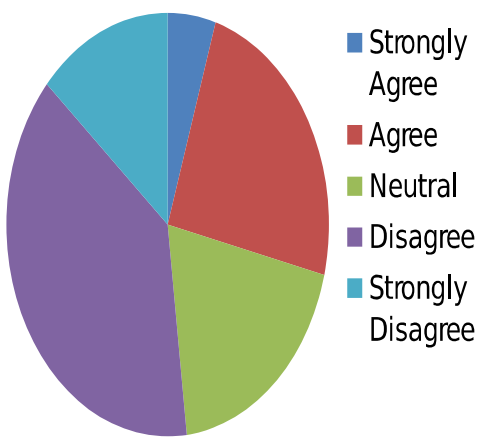

Has your organization ever utilized the benefits of lifi?

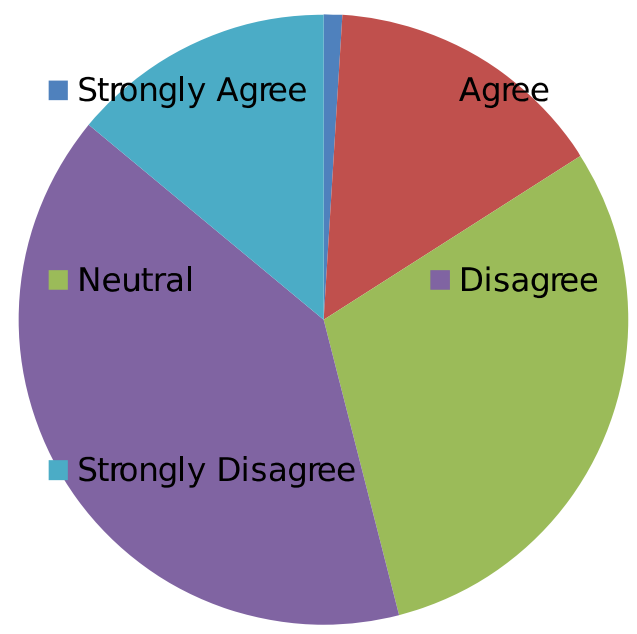

Are you aware of the lifi technology?

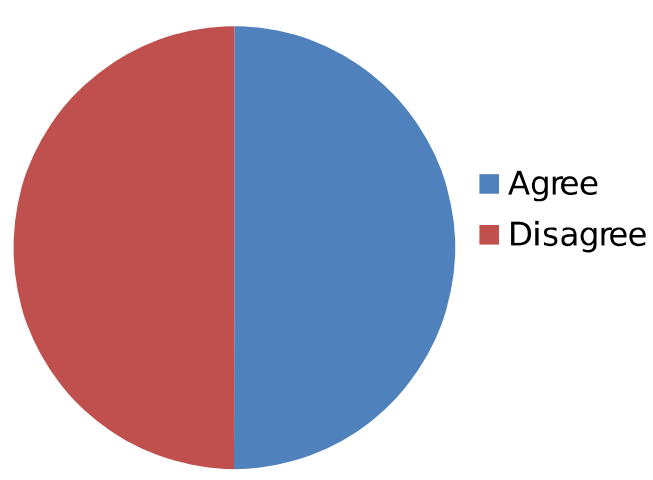

Are you aware of any other firm that has been utilizing the lifi technology?

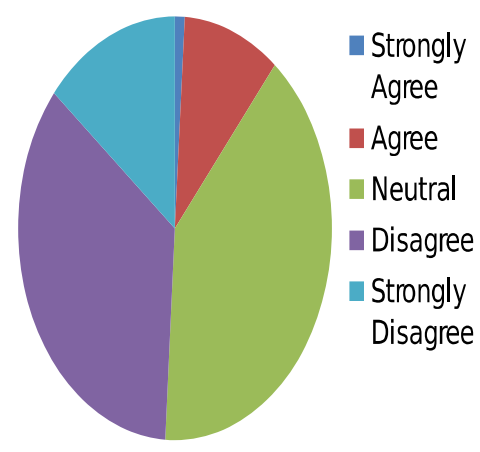




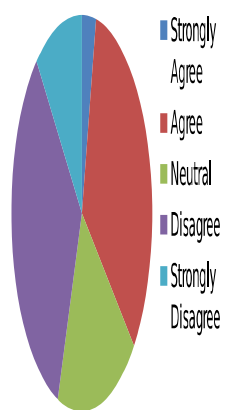

\section{CONCLUSION}

LI-FI is a rising innovation and subsequently it has incomprehensible potential. This innovation can get to be one of the real advancements sooner rather than later. On the off chance that this innovation can be utilized proficiently, clients may soon have something of the sort of WI-FI hotspots wherever a light is accessible.

$\mathrm{Li}-\mathrm{Fi}$ is the upcoming and on developing innovation going about as equipped for different other creating and right now designed advancements. Since light is the real hotspot for transmission in this innovation it is extremely worthwhile. Henceforth the future uses of the Li-Fi can be anticipated and reached out to various stages like training fields, therapeutic field, mechanical ranges and numerous other fields.[14]

The eventual fate of LI-FI is GI-FI. GI-FI or gigabit remote alludes to remote correspondence at an information rate of more than one billion bits (gigabit) every second. In 2008 analysts at the University of Melbourne exhibited a handset coordinated on a solitary incorporated circuit (chip) that worked at $60 \mathrm{GHz}$ on the CMOS process. It will permit remote exchange of sound and video information at up to 5 gigabits for every second, ten times the present most extreme remote exchange rate, at one-tenth the expense. Scientists picked the 57-64 GHz unlicensed recurrence band subsequent

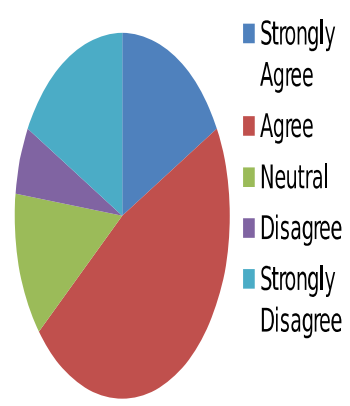

to the milli meter-wave scope of the range permitted high segment on-chip coordination and in addition the reconciliation of little high pickup exhibits. The accessible $7 \mathrm{GHz}$ of range results in high information rates, up to 5 gigabits for each second to clients inside of an indoor domain, more often than not inside of a scope of 10 meters. It's evaluated that the Li-Fi business sector will be worth more than $\$ 6$ billion by 2018, as indicated by examination by MarketsandMarkets.[16]

There positively is space for $\mathrm{Li}-\mathrm{Fi}$ and other unmistakable light-based correspondence frameworks to develop in corner zones; however switch makers can breathe a sigh of relief for some time.

\section{REFERENCES}

[1] Kanchan Gupta, Kajal, Ashish Saini, "Light Fidelity TechnologyA Review," International Journal of Research (IJR), Vol-1, Issue-10 pp. 135-139, November 2014.

[2] Sharma, R. R., \&Sanganal, A. (2014). Li-Fi Technology: Transmission of data through light. International Journal of Computer Technology and Applications, 5(1), 150.

[3] Swanson, M. (2001). Security selfassessment guide for information technology systems (No. NIST-SP- 
800-26). BOOZ-ALLEN AND HAMILTON INC MCLEAN VA.

[4] Singh, S. Prof.(Dr.) YP "LI FI-A NEW PARADIGM" International Journal of Advanced Research in. Computer Science and Software Engineering, ISSN,2277.

[5] Rawat, B. S., Aggarwal, B., \&Passi, D. (2014). LI-FI: A new era of wireless communication

data sharing. IJSTR, 3, 118-119.

[6] Rani, J., Chauhan, P., \&Tripathi, R. (2012). Li-Fi (Light Fidelity)-The future technology In Wireless communication. Int. J. of Applied Engineering Research, 7(11).

[7] Jitender Singh, Vikash "A New Era in Wireless Technology using LightFidelity" International Journal of Recent Development in Engineering and Technology ISSN 23476435(Online) Volume 2, Issue 6, June 2014

[8] R.Karthika, S.Balakrishnan "Wireless Communication using Li-Fi Technology" SSRG International Journal of Electronics and Communication Engineering (SSRGIJECE) volume 2 Issue 3 March 2015 [9] Dinesh Khandal, Sakshi Jain "Li-Fi (Light Fidelity): The Future Technology in Wireless Communication" International Journal of Information \& Computation Technology. ISSN 09742239 Volume 4, Number 16 (2014) [10]Qian Huang, Xiaohang Li, Mark Shaurette "Integrating Li-Fi Wireless Communication and Energy Harvesting Wireless Sensor for Next Generation Building Management" International High Performance Building Conference, Purdue University.

[11]Ekta, Ranjeet Kaur Light "Fidelity (LI-FI)-A Comprehensive Study" International Journal of Computer
Science and Mobile Computing Vol. 3, Issue. 4, April 2014, pg.475 - 481 ISSN 2320-088X

[12] Nivrutti, D. V., \&Nimbalkar, R. R. (2013). Light-Fidelity: A Reconnaissance of Future Technology. International Journal of Advanced Research in Computer Science and Software Engineering, 3(11), 753-756.

[13] Bhut, J. H., Parmar, D. N., \& Mehta, K. V. (2014). LI-FI TechnologyA Visible Light Communication.

[14] Navyatha, N., Prathyusha, T. M., Roja, V., \&Mounika, M. (2013). Li-Fi (Light fidelity)-LED Based Alternative. International Journal of Scientific \& Engineering Research, 4(5), 1039-1042.

[15] Tanwar, K., \& Gupta, S. (2014). Smart Class Using Li-Fi Technology.International Journal of Engineering and Science (IJES), 3(7). [16] Paul, S., \& Sharma, S. (2014). Future of Telecommunication Technologies: Wi-fi vs. Wi-max vs. Li-fi vs. Gi-fi. ISTP Journal of Research in Electrical and Electronics Engineering, 128-136.

[17] Virk, G. K. (2015). Li-Fi: A New Communication

Mechanism. International Journal of Computer Applications, 118(15)

[18] Aggarwal, A., \&Jhanji, D. (2014). Comparative study: Li-Fi v/s WiFi.International Journal of Research \& Development in Technology and Management Science, 21(1).

[19] Chatterjee, S., Agarwal, S., \&Nath, A. scope and Challenges in Light Fidelity (LiFi) Technology in Wireless Data Communication. International Journal of Innovative Research in Advanced Engineering (IJIRAE), (6), 1-9. 